\title{
Whole genome analysis of ExPEC ST73 from a single hospital over a 2-year period identified different circulating clonal groups
}

Bogema DR ${ }^{1,2} *$, McKinnon $\mathrm{J}^{2 *}$, Liu $\mathrm{M}^{2}$, Hitchick $\mathrm{N}^{3}$, Miller $\mathrm{N}^{3}$, Venturini $\mathrm{C}^{4}$, Iredell, $\mathrm{J}^{4,5}$, Darling $\mathrm{AE}^{2}$, Roy Chowdury $\mathrm{P}^{2}$, Djordjevic $\mathrm{SP}^{2} \%$

${ }^{1}$ Elizabeth Macarthur Agricultural Institute, NSW Department of Primary Industries, Menangle, NSW, 2568 Australia.

${ }^{2}$ The ithree Institute, University of Technology Sydney, NSW, 2007 Australia.

${ }^{3}$ San Pathology, Sydney Adventist Hospital, Wahroonga, NSW, 2076 Australia

${ }^{4}$ Centre for Infectious Diseases and Microbiology, The Westmead Institute for Medical Research, The University of Sydney, Westmead, NSW, 2145 Australia

${ }^{5}$ Westmead Hospital, Westmead, NSW, 2145 Australia

* authors contributed equally to this manuscript

† corresponding author; Email: steven.djordjevic@ uts.edu.au; Ph. +61-2- 9514-4127

Keywords: Uropathogenic Escherichia coli, class 1 integron, 


\section{$\underline{\text { Abstract }}$}

ST73 has emerged as one of the most frequently isolated extraintestinal pathogenic E. coli (ExPEC). To examine the localised diversity of ST73 clonal groups including their mobile genetic elements profile, we sequenced the genomes of 16 multiple drug-resistant ST73 isolates from patients with urinary tract infection from a single hospital in Sydney, Australia between 2009 and 2011. Genome sequences were used to generate a SNP-based phylogenetic tree to determine the relationship of these isolates in a global context with ST73 sequences $(n=210)$ from public databases. There was no evidence of a dominant outbreak strain of ST73 in patients from this hospital, rather we identified at least eight separate groups, several of which reoccur, over a two-year period. The inferred phylogeny of all ST73 strains $(n=226)$ including the ST73 Clone D i2 reference genome shows high bootstrap support and clusters into four major groups which correlate with serotype. The Sydney ST73 strains carry a wide variety of virulence-associated genes but the presence of iss, pic and several iron acquisition operons was notable.

\section{Impact}

ST73 is a major clonal lineage of ExPEC that causes urinary tract infections often with uroseptic sequelae but has not garnered substantial scientific interest as the globally disseminated ST131. Isolation of multiple antimicrobial resistant variants of ExPEC ST73 have increased in frequency, but little is known about the carriage of class 1 integrons in this sequence type and the plasmids that are likely to mobilise them. This pilot study examines the ST73 isolates within a single hospital in Sydney Australia and provides the first large-scale core-genome phylogenetic analysis of ST73 utilizing public sequence read datasets. We used this analysis to identify at least 8 sub-groups of ST73 within this single hospital. Mobile genetic elements associated with antibiotic resistance were less diverse and only three class 1 
integron structures were identified, all sharing the same basic structure suggesting that the acquisition of drug resistance is a recent event. Genomic epidemiological studies are needed to further characterise established and emerging clonal populations of multiple drug resistant ExPEC to identify sources and aid outbreak investigations.

\section{$\underline{\text { Introduction }}$}

Extraintestinal pathogenic Escherichia coli (ExPEC) are phylogenetically diverse and comprise uropathogenic E. coli (UPEC), neonatal meningitis-causing E. coli (NMEC) and avian pathogenic E. coli (APEC). ExPEC account for $\sim 75-95 \%$ of urinary tract infections (UTI). A proportion of these infections can spread from the urinary tract with invasion of epithelial cells in the bladder (cystitis) and kidney cells (pyelonephritis), and transmission to systemic circulation (blood sepsis), posing a serious threat to human health. ExPEC are enteric bacteria but their capacity to capture a wide array of virulence-associated genes (VAGs) by lateral gene transfer has expanded the repertoire of niches they colonise. ExPEC may carry diverse and often redundant combinations of VAGs whose impact on human health remains ill-defined. Epidemiological studies indicate that a subset of pathogenic E. coli lineages including ST73, ST131, ST405, ST393, ST69, ST95, ST10, ST38, and ST127 [1-5] are responsible for most ExPEC infections [3, 6-8]. Carriage of combinations of virulence genes enhance virulence [9], however, carriage of antimicrobial resistance genes, particularly those encoding ESBLs and fluoroquinolones, as well as an ability to cause opportunistic infections in vulnerable (elderly) hosts, may also contribute to virulence. It is notable that none of these hypotheses have been experimentally validated $[3,6]$.

ExPEC have become the leading cause of blood sepsis in Europe [10]. Notable in this regard is the alarming rise in the incidence of ST73, now one of the most frequently isolated UPEC globally and the leading cause of bacteraemia in the East Midlands region of the UK $[1,11$ - 
14]. ST73 belongs to Clermont phylogroup B2 and is known to display different serogroups with serotype O6 predominating (ST73-O6-B2) $[15,16]$. It has recently been suggested that the rise in the incidence of multiple drug resistant (MDR) ST73 in the UK is not due to the emergence of a dominant clone because they are genetically diverse and carry a different array of plasmids encoding resistance to multiple antimicrobials. Many recently described isolates of ST73 carry genes that encode extended spectrum $\beta$-lactamases and resistance to antimicrobials used in veterinary medicine [14]. This seems to be a recent adaptation in this ST as previously characterized ST73 isolates from cases of uncomplicated UTI sourced from Greece, Portugal, Sweden and the UK, resulted susceptible to most clinically relevant antimicrobials [17] and most (75\%) did not carry plasmids, classic vehicles of MDR [17]. These data combined with the most recent findings seem to suggest that the rise in the carriage of MDR plasmids in ST73 may be a recent concerning event $[14,18]$.

Here we have characterised whole genome sequences of 15 class 1 integrase (intI1)containing ST73 strains from a hospital in Sydney. To determine if these highly localised strains were from a limited number of clonal lineages, phylogenetic inferences were made by comparing SNP differences in core genomes shared by ST73 strains from our Sydney collection with those from six high-quality reference genome sequences and ST73 strains $(n=204)$ from seven countries sourced from global sequence read archives. We also examined mobile and chromosomal genetic content within this localised isolate cohort to further examine their diversity. We compiled the repertoire of antimicrobial genes and virulence genes and mapped the class 1 integron structures carried by these isolates. As carriage of the class 1 integrase is considered a reliable proxy for multiple drug resistance [19], we used S1PFGE followed by Southern hybridization with an intIl probe to examine plasmid content and carriage of the class 1 integrase on plasmids. 


\section{Methods}

\section{Isolate source and culture conditions}

Clinical samples in this project were from a larger collection obtained from Sydney Adventist Hospital from 2009-2011. Bacterial species were identified by the VITEK® 2 (bioMérieux) system at Sydney Adventist Hospital. For DNA extraction, strains were first grown on a Lysogeny Broth (LB) agar plate to isolate single colonies of which one was used to inoculate $2 \mathrm{~mL}$ of LB broth followed by shaking for 16 hours at $37^{\circ} \mathrm{C}$. Antibiotic susceptibility testing for ampicillin, cefotaxime, chloramphenicol, streptomycin and sulfafurazole was performed via the CDS method [20]. These antibiotics were selected based on antibiotic resistance gene content inferred from genome sequencing.

\section{Nucleic acid purification and whole genome sequencing}

E. coli DNA was extracted using the Isolate II Genomic DNA Extraction kit (Bioline) according to the manufacturer's instructions. For each sample, tagmentation of genomic DNA, and PCR amplification of tagged DNA were performed in triplicate using the Nextera system (Illumina). Sequencing libraries were pooled, then cleaned and size selected using SPRI beads (Beckman Coulter). Normalization was guided by read counts obtained from a Nano flowcell run on a MiSeq instrument. Agilent 2100 Bioanalyzer, with High Sensitivity DNA kit was used to quantitate the pooled library before loading onto an Illumina HiSeq. Paired-end $150 \mathrm{bp}$ reads were generated using the HiSeq 2500 v4 system.

\section{Genome assembly and gene presence}

Genome assembly was achieved with raw reads using the A5-miseq pipeline [21] and checked for consistency by additional assembly with SPAdes 3.9.0 [22]. Antibiotic resistance and virulence-associated genes were identified from assembled genomes using BLASTn and 
SRST2 [23]. Searches were performed against antibiotic resistance genes sourced from the ARG-ANNOT V3 database and a panel of virulence-associated genes identified from the Virulence Factors[24] Database (VFDB) and literature searches [25]. Serotyping was performed in silico with SRST2 using EcOH sequences supplied with this package. Draft genome reads obtained from SRA were searched for a minimal set of marker genes derived from integron structures characterised in the Sydney strains. Low-quality alignments based on SRST2 output were discounted $(n=3)$.

\section{Archived sequence read selection}

All additional ST73 sequences not generated by this study were obtained from complete whole genome assemblies $(n=6)$ [26-30] and public sequence read archives (NCBI-EMBL). Raw Illumina reads sourced from ST73 isolates $(n=284)$ were considered for SNP-based phylogenetic analysis, including strains sequenced in this study from the Sydney Adventist Hospital $(\mathrm{n}=16)$, isolates with host, source and isolation location meta-data identified from the EnteroBase database ( $\mathrm{n}=246$; http://enterobase.warwick.ac.uk/; accessed 5/12/2016) and a previous ST73-focused study from the United Kingdom $(n=22)$ [11]. Samples were excluded if sequence type could not be confirmed as ST73 using SRST2 $(n=4)$. Further samples were excluded $(n=30)$ if isolate status could not be confirmed by BioProject metadata or where the description of methods could not be identified by an associated publication [31-38]. Samples were additionally excluded $(n=30)$ if they produced low reference genome coverage (>90\%) in whole-genome alignments. Additional sample filtering of ST73 reads is described in Table S2.

\section{S1-PFGE analysis}

The complement of large (>20 kb) plasmids in each bacterial isolate was determined by S1 nuclease (Promega, Madison, WI, USA) digestion and pulsed-field gel electrophoresis 
(PFGE) as described previously [39, 40]. Southern blot hybridization was used to determine the genomic location of the intIl gene. PCR amplicons for intIl were obtained using published primers (int1F and int1R; [41]) and labelled using the PCR DIG Probe Synthesis Kit (Roche, Mannheim, Germany). DNA was transferred from the S1-PFGE gel to a nylon membrane (GE Health, Little Chalfont, UK) using a VacuAid vacuum transfer apparatus (HybAid, Teddington, UK) and hybridisation was performed using the Roche DIG Filter Hybridization system (Roche, Mannheim, Germany) following manufacturer's instructions. Images were acquired on a ChemiDoc ${ }^{\mathrm{TM}}$ MP System (Bio-Rad Laboratories, Richmond, CA, USA).

SNP based phylogenetic analyses

Our initial attempts to examine ST73 phylogeny with our 16 genome sequences and 6 complete whole genome sequences (CFT073, ABU83972, ATCC25922, Nissle 1917, clone D i2 and clone D i14) using marker gene approaches [42] provided limited resolving power (Fig. S1). Consequently, to more appropriately examine these ST73 strains, we employed SNP-based phylogenetic methods.

For SNP-based phylogenetic trees, core genome alignments were generated with Snippy v3.1.0 (https://github.com/tseemann/snippy) using default options. Briefly, reads were mapped using bwa mem v0.7 to an ST73 reference genome. Raw alignments were processed with samtools v1.3.1 and variants called using freebayes v1.1.0. SNP derived genomes were reconstructed using vcftools v0.1.14, with low-coverage $(<10 \mathrm{x})$ and degenerate reference positions filtered. Recombinant regions were removed using Gubbins v2.20 (options -i 10) [43] yielding aligned, SNP-derived, recombination-filtered core genomes. From this alignment, core genome phylogenetic trees were inferred by maximum-likelihood using RAxML v8.2.9 [44]. Branch support was estimated by bootstrap analysis employing 100 
bioRxiv preprint doi: https://doi.org/10.1101/428599; this version posted September 27, 2018. The copyright holder for this preprint (which was not certified by peer review) is the author/funder, who has granted bioRxiv a license to display the preprint in perpetuity. It is made available under aCC-BY-NC-ND 4.0 International license.

Temporal analysis of $E$. coli ST73

replicate trees. Trees were rooted using the ultrametric tree method included with RAxML. Scripts used for the analysis of SNP phylogeny can be found online at https://github.com/bogemad/snp_phylogeny.

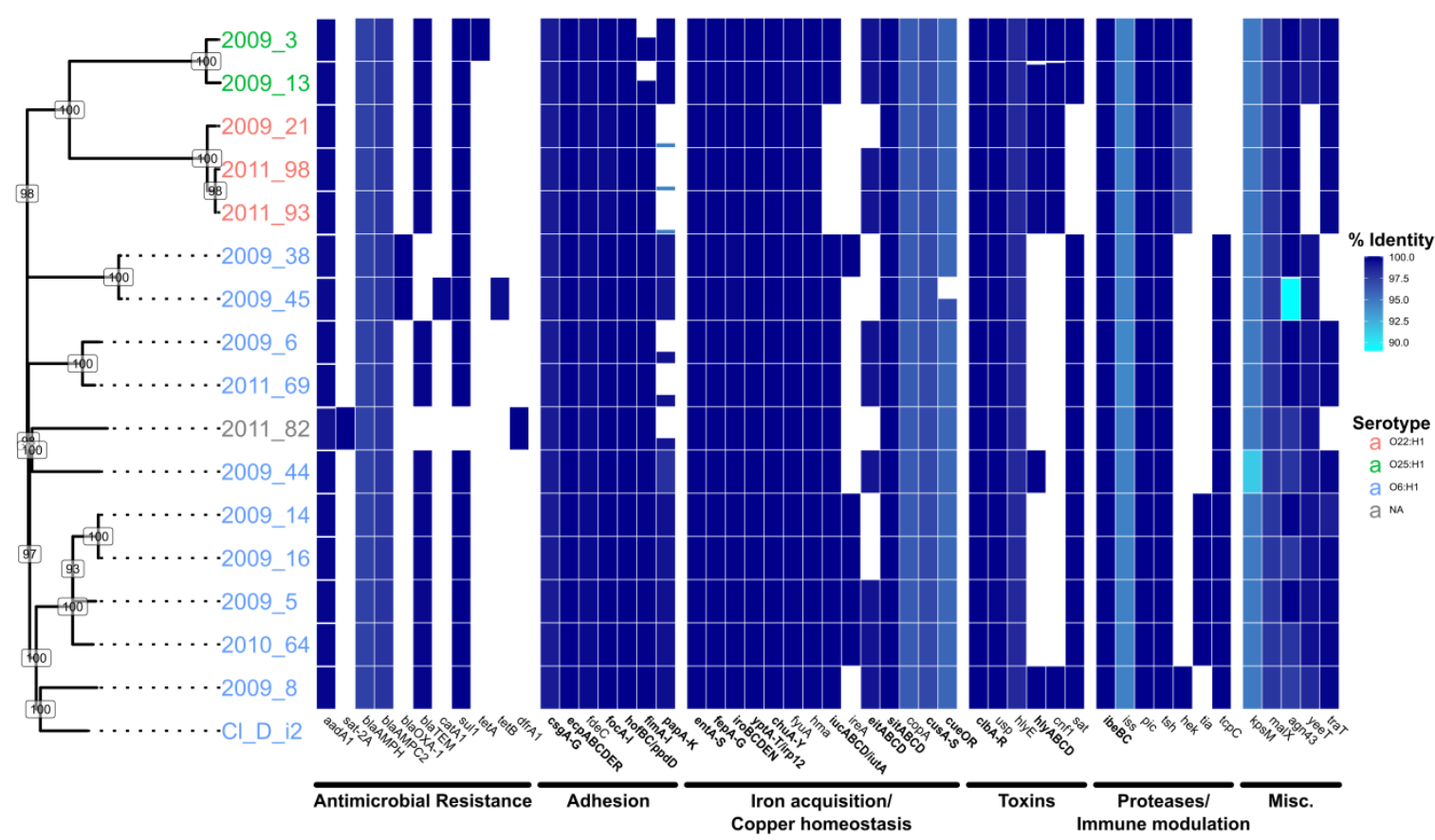

Figure 1. A SNP-dervied phylogenetic tree of the Sydney ST73 strains sequenced in this study compared with antimicrobial resistance and virulence profiles. Bootstrap values based on 100 replicate trees are shown at labeled nodes. Isolate serotypes as determined by in silico serotyping are shown by coloured tip labels. Antibiotic resistance and virulence gene/genefamily presence (blue) or absence (white) is shown by the linked bar-graph/heatmap. Percentage identity of BLAST matches is indicated by heatmap shade with darker shades representing higher identity. BLAST match coverage is represented by tile height with solid tiles representing 100\% coverage. For gene families (x-axis; bold) tile height represents total BLAST match coverage of all gene family members and shows completeness of the gene family. Where single genes are indicated (x-axis; plain text), bar height represents BLAST match coverage of the gene.

Initially we performed this analysis using only isolates sequenced in this study and a highquality published ST73 reference (Fig. 1). To identify the most suitable reference genome for this purpose, we aligned reads individually to the six complete reference genomes (above) and examined reference sequence quality, core alignment lengths and final tree support values. Using this methodology, the most suitable reference was identified as clone D i2 
which with archived public sequence reads generated a core genome of 3,818,344 bp, representing $75.8 \%$ of the ST73 clone D i2 sequence.

\section{$\underline{\text { Results }}$}

\section{Assembly information and statistics}

The genome sequences of 16 ST73 strains from the Sydney Adventist Hospital were determined here. The Whole Genome Shotgun project has been deposited at DDBJ/ENA/GenBank and the sequence read archive. Assembly statistics as well as accession numbers, number of sequencing reads, and amount of sequencing data used to generate assemblies can be found in Table $\mathrm{S} 1$.

\section{Public read high-throughput sequencing analysis}

The Sydney isolates separated in several groups that closely aligned with E. coli serotype including O22:H1, O25:H1 and O6:H1. However, most of the Sydney isolates clustered within a larger O6:H1 group. To further interrogate observed diversity within the O6:H1 group of isolates and to place Sydney strains within a broader global context, we expanded the SNP-based phylogenetic analysis to include additional ST73 sequence reads obtained from public sequence read archives (NCBI-EMBL-DDBJ) and the aforementioned six complete ExPEC genomes with the 16 Sydney genomes. The inferred phylogeny of the 226 strains (Fig. 2; see Fig. S2 for all strain labels and branch support values) shows strong major branch support. Analysis of the SNP-derived phylogenetic tree shows correlation with observed serotypes $\mathrm{O} 6: \mathrm{H} 1, \mathrm{O} 25: \mathrm{H} 1$ and $\mathrm{O} 22: \mathrm{H} 1$, with most strains observed within the O6:H1 cluster. Strain 2011_82 could not be assigned an O-type from in silico serotyping but was identified as H1 and clustered most closely with O6:H1 isolates (Fig. 2 - Serotype). 


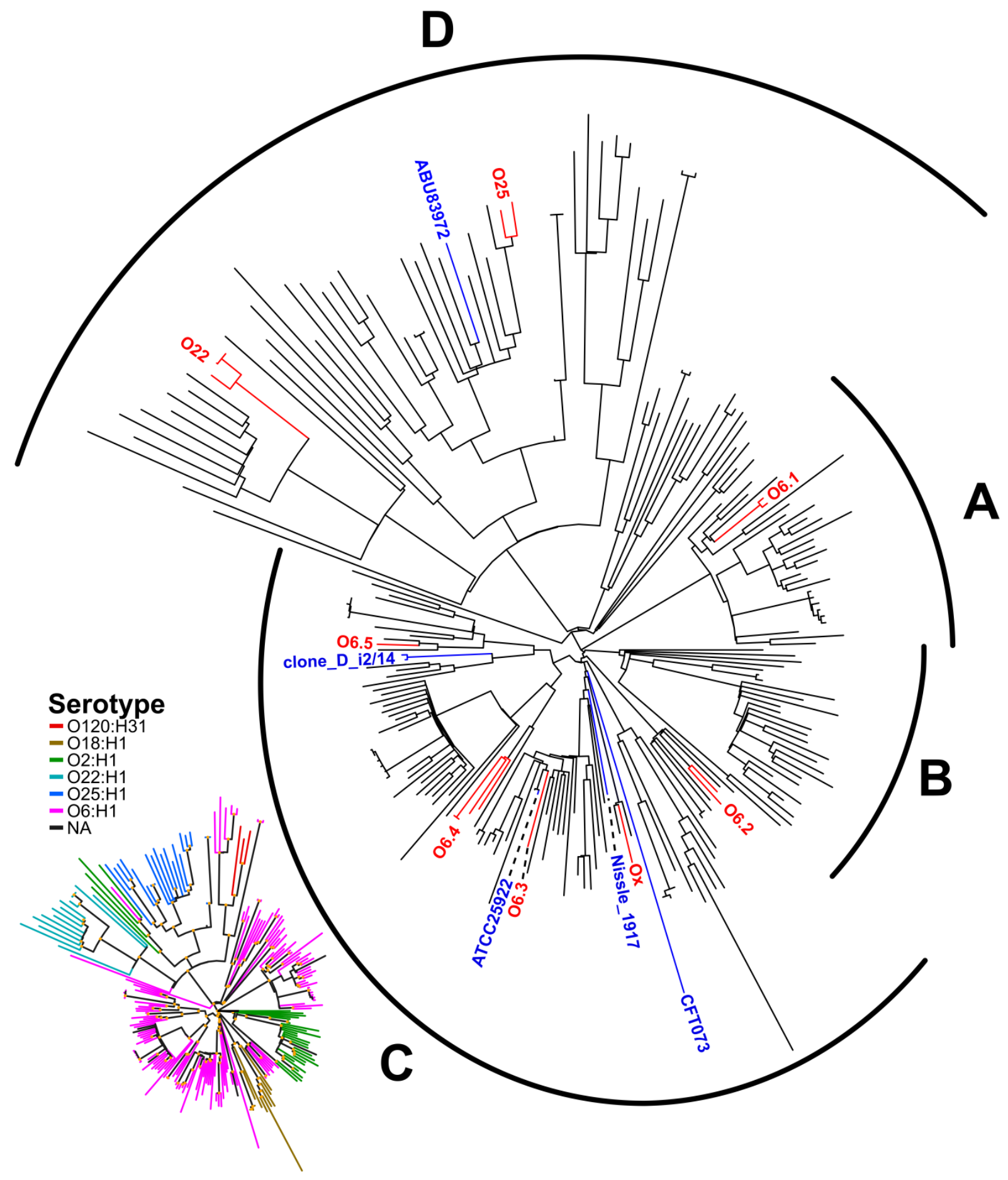

Figure 2. SNP-based maximum-likelihood phylogram of 226 ST73 strains. A more detailed tree with branch support values and tip labels can be found in Figure S2. ST73 isolates separate into 4 distinct groups, labelled A-D, which correlate well with in silico serotyping (insert). ST73 isolates sequenced in this study cluster into 8 distinct groups, shown in red, high-quality complete ST73 genomes are shown in blue. Trees were constructed using 18,426 SNPs identified by read mapping to the clone D i2 reference sequence, reduced from 27,568 SNPs by filtering of recombination regions. 
Examination of ST73 phylogenetic structure reveals four significant clades (Fig. 2 and S2): one exclusively associated with serotype O6:H1 (Group A), one exclusively associated with serotype O2:H1 (Group B), one primarily serotype O6:H1 with a subclade of O18:H1 (Group C), and finally a polyphyletic group consisting of $\mathrm{O} 2: \mathrm{H} 1, \mathrm{O} 6: \mathrm{H} 1, \mathrm{O} 22: \mathrm{H} 1, \mathrm{O} 25: \mathrm{H} 1$ and O120:H31 serotypes (Group D). Strains sequenced from Sydney separated into eight distinct groups correlated with serotype (Fig. 2 - red labels). Isolates with O22:H1 and O25:H1 serotypes formed their own clusters, while serotype O6:H1 separated into 5 distinct clusters (O6-1: 2009_38/45; O6-2: 2009_6/2011_69; O6-3: 2009_44; O6-4: 2009_5/14/16/2010_64; O6-5:2009_8). Strain 2011_82 clustered with other O6:H1 strains and formed the final group $(\mathrm{Ox})$.

\section{Virulence profiles of Sydney strains}

We identified differences in virulence gene profiles of E. coli strains examined in this study (Fig. 1). Significantly, we found that virulence gene profiles were largely consistent in strains from the same phylogenetic groups O25, O22, O6-1 - O6-5, Ox (Fig. 1, see Table S4 and S5 for greater detail). For adhesion-related genes, critical components of $\mathrm{P}$ fimbriae (papACDEFGHJK) were absent in six strains (Fig. 1). Additionally, in strains of group $\mathrm{O} 25$, genes encoding the Type I fimbriae major subunit (fimA), periplasmic chaperone $(\operatorname{fim} C)$, regulatory subunit (fimE) and the fimbriae-associated fimI were missing in blastn searches. Other genes encoding F1C fimbriae, Curli fibres, Type IV pili, E. coli common pili and the $f d e C$ adhesion genes were present in all strains. The importance of FdeC as a putative virulence factor is underpinned by the observation that it is i) a broadly conserved, E. coli adhesin whose expression is upregulated on the surface of UPEC when it contacts host cells; and ii) a major target during humoral immune responses that significantly reduced kidney colonization in mice challenged transurethrally with UPEC strain 536 [45]. 
Iron acquisition is critical for the growth of ExPEC in low iron environments in vivo and it is not uncommon to identify genes linked to siderophore production and processing in UPEC. Complete Enterobactin, Salmochelin, Yersiniabactin gene clusters were identified in all ST73 strains while Aerobactin genes were identified in all strains except those belonging to group O22. Genes for heme uptake including the chu operon and hma gene were present in all strains, as were those related to iron uptake such as the sit $\mathrm{ABC}$ transporter operon and ferric Yersinia uptake $(f y u A)$ gene. In contrast, the putative iron uptake gene cluster eitABCD and adhesion/iron-uptake gene ireA were only identified in a subset of strains. In addition to iron uptake, genes encoding copper resistance have also been linked to virulence [46] and antimicrobial resistance [47]. The cus system, encoding a four-component copper efflux pump, was present and complete in all strains. However, in strain 2009_45, cueR, an important regulator controlling copper detoxification and efflux copA and cueO genes, was not located in all searches.

Larger differences were observed in the presence of toxin genes. Strains from group O25 and strain 2009_8 contained the highest number of toxin genes including cytotoxic necrotizing factor 1 (cnfl), the hemolysin (hlyABCD) cluster, hemolysin $\mathrm{E}$ (hlyE) and secreted autotransporter toxin (sat). Genes that have been previously shown to promote propagation of E. coli in blood, such as proteases pic and tsh and the increased serum survival (iss) gene were present in all strains as well as cellular invasion promoting ibe gene cluster. Closely related hek and tia genes, associated with epithelial cell invasion in Neonatal Meningitiscausing and enterotoxigenic E. coli respectively, are both found in separate strains. Furthermore, tcpC associated with immune modulation via inhibition of Toll/interleukin-1 (IL-1) receptor signalling, was only found in groups O6-1-5 and Ox. 
Temporal analysis of E. coli ST73

\section{Antibiotic resistance}

All intIl positive isolates were tested for resistance to ampicillin, cefotaxime, chloramphenicol, streptomycin, sulfafurazole and trimethoprim using the CDS method (Table 1). Strain 2011_82 did not have a class 1 integrase gene and was not tested. All strains were resistant to ampicillin, streptomycin and sulfafurazole. Genes encoding resistance to these antibiotics were all accounted for in the genome sequence data by the class 1 integronassociated genes aadAl and sull as well as one of three bla gene variants. Only strain 2009_45 was resistant to the third-generation cephalosporin cefotaxime, likely due to the presence of the bla OXA-1 $_{1}$ gene. However, this resistance was not observed in strain 2009_38 which contained an almost identical antimicrobial resistance region, suggesting this gene is not expressed in this strain. Interestingly, both of these strains also showed phenotypic resistance to chloramphenicol despite only 2009_45 containing a complete copy of the catA1 gene. The full repertoire of antibiotic resistance genes found in the 16 Sydney ST73 strains is presented in Figure 1.

Table 1: Phenotypic antimicrobial resistance

\begin{tabular}{lcccccccc} 
Patient & AMP & AMC & LEX & CIP & GEN & NIT & NOR & TMP \\
\hline $2009 \_3$ & R & R & S & S & S & S & S & S \\
$2009 \_5$ & R & R & S & S & S & S & S & S \\
$2009 \_6$ & R & R & S & S & S & S & - & S \\
$2009 \_8$ & R & S & S & S & S & S & - & S \\
2009_13 & R & S & S & S & S & S & S & S \\
2009_14/16 & R & S & S & S & S & S & - & S \\
2009_21 & R & S & S & S & S & S & - & S \\
2009_38 & R & S & S & S & S & S & S & S \\
2009_44 & R & - & S & S & S & S & S & S \\
2009_45 & R & S & S & S & S & S & S & S \\
2010_64 & R & S & S & S & S & S & - & S \\
2011_69 & R & S & S & S & S & R & S & S \\
2011_82 & S & - & S & S & S & S & S & R \\
2011_93/98 & R & S & S & S & S & S & - & S
\end{tabular}


Structure of class 1 integrons in ST73 strains from Sydney

All locally sourced strains in this study, excepting 2011_82, were positive for a complete copy of the sulfonamide resistance gene sull, a structural marker of the $3^{\prime}$-conserved segment (3'-CS) of class 1 integrons. Similarly, all strains contained the aminoglycoside resistance gene cassette aadA1. Strain 2011_82 was found to contain only a class 2 integron carrying the standard $d f r A 1$-sat2-aadAl cassette array.

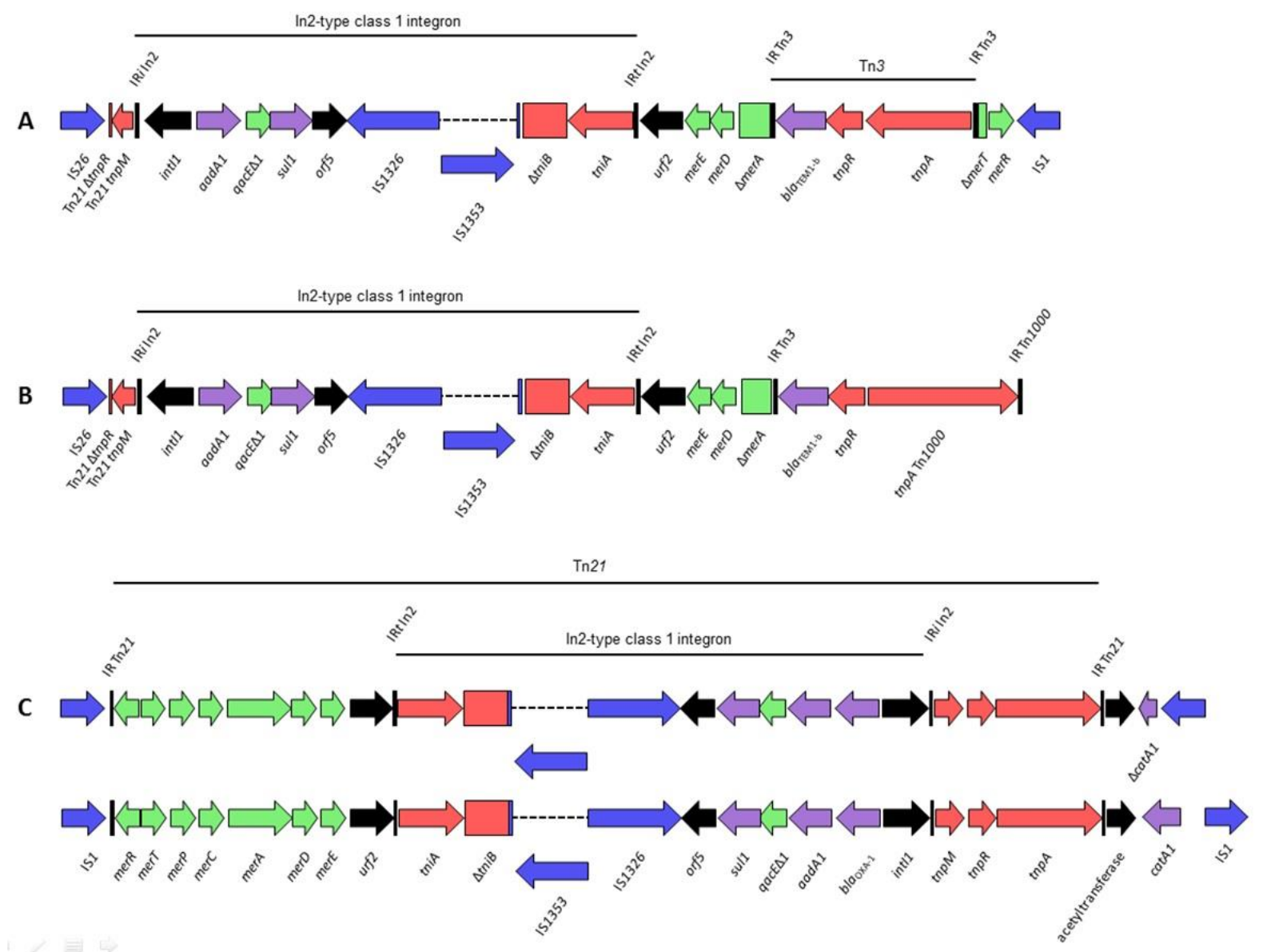

Figure 3. Schematic representations of integron structures found within this collection.

There were three class 1 integron-containing resistance regions represented within our collection (Fig. 3), all containing the same base structures with minor variations. The first structure was identified in 11 out of 15 class 1 integron-containing isolates (Fig. 3A). It consisted of an In-2 type class 1 integron with and aadAl gene cassette housed within an 
incomplete $\operatorname{Tn} 21$ transposon, matching (99\% sequence identity) to the sequence in the R100 plasmid identified in Japan in the 1950s from Shigella flexneri Accession NC_002134.1 [48]. However, our structure bears an IS26-mediated partial deletion of the Tn21 tnpR gene, which is a signature that has been reported previously twice within a uropathogenic E. coli strain from Australia, and in association with a different class 1 integron structure [49]. A Tn3 transposon has inserted within the mer module of $\operatorname{Tn} 21$ with partial deletion of merA and merT and complete deletion of merC and merP. The transposon is abutted downstream of merR by an inward facing IS 1 insertion element. One strain, 2009-64, housed this exact structure apart from the Tn21 tnpM, which appears to have been lost due to an IS26-mediated deletion event.

The complex resistance locus (CRL) shown in Figure 3B was identified in isolates 2009-6 and 2011-69, and shares homology with the structure in Figure 3A. It bore identical IS26 and Tn3 insertion points, with the only major difference being a crossover event where the standard $\operatorname{Tn} 3 \operatorname{tnp} A$ gene and terminal inverted repeat have been replaced by that of $\operatorname{Tn} 1000$, a transposon originally identified in a cosmid clone of a human DNA sequence in 1995 [50]. This signature was recently identified in the sequence of an unannotated plasmid of a Salmonella enterica serovar Typhi strain sequenced as part of a larger study of Typhi from typhoid-endemic regions of Asia and Africa (Accession LT904889.1). This is therefore the first report of this hybrid transposon and its presence in an E. coli isolated in Australia. Due to the nature of Illumina sequence technology we have no confirmed sequence information downstream of $\operatorname{Tn} 3 / \operatorname{Tn} 1000$.

Structure 3 (Fig. 3C) shares homology to the previously discussed structures. However, this

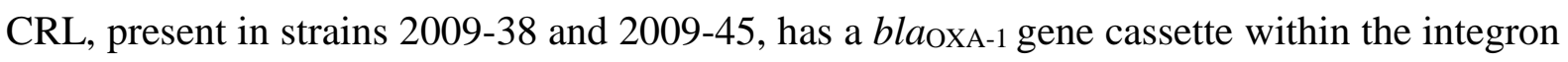
cassette array in addition to aadA1. Here, the Tn21 transposon housing the class 1 integron is complete, with both the initial and terminal inverted repeats intact, and has an inward facing 
IS1 flanking its mer end. There are two variants of this CRL in our collection, one of which contains a complete catAl gene downstream of Tn21 followed by a second IS 1 element in the same orientation as the first, with the intergenic ORF identified as an acetyltransferase. This appears to be an established insertion event, with numerous reports in GenBank. In the second variant, the terminal IS 1 is inverted with consequent deletion of $476 \mathrm{bp}$ of the catA 1 gene, forming a signature unique to isolates 2009-38 and 2009-45. This integron has been reported in its entirety in Shigella dysenteriae 1 plasmid p3099-85 (KT754164.1), Salmonella enterica serovar Typhimurium plasmid pUO-StVR2 (AM991977.1) and Salmonella enterica serovar Typhimurium strain T000240 (AP011957.1) Less than 10 SNPs were identified in comparative sequence alignments spanning the integron.

Sixty genomes from the SRA cohort returned adequate alignments to integron marker genes, with 25 of these appearing to have only the base class 1 integron with an aadAl cassette but no indication of a bordering Tn21 transposon. Eight contained an intIl gene but no aadAl, suggesting the presence of a class 1 integron with a different cassette array. Sixteen contained aadAl but no class 1 integrase; this could indicate a deletion event or more likely the presence of aadAl in a class 2 integron context. Five genomes contained an unidentifiable integron structure, possibly variants of those described in the Sydney collection though it is impossible to say this definitively from read alignments against the abridged gene database used here.

Only three genomes; HVH_93_4-5851025, MOD1-EC6690 and MOD1-EC6783, contained all marker genes necessary to potentially contain integron C (Figure 3). However, within the SRA cohort, the presence of integrons A and B could not be confirmed. 
All 16 strains of ST73 that were sourced from Sydney were shown to carry one or more plasmids (up to five) that ranged in size from 15 to $180 \mathrm{~kb}$. Only one plasmid in each strain hybridized with the intIl probe (data not shown).

\section{$\underline{\text { Discussion }}$}

This study forms a part of wider global efforts to further understand the structure of diseasecausing ST73 clones. Whole genome sequencing and maximum-likelihood phylogenetic analyses of these clones is providing important information on the community structure of ExPEC. Here we examined 16 ST73 isolates sourced from a single hospital and used sequence data sourced from the Sequence Read Archive to place these isolates into a broader global context and aid in identifying clonal lineages. Phylogenetic trees from this combined dataset, when overlaid with geographical and temporal data sourced from EnteroBase (data not shown), indicate that ST73 is globally disseminated in a manner similar to ST131 [51, 52] which is currently the most studied pandemic ExPEC lineage due to the frequency of CTX-M gene carriage. However, while ST131 tends to be relatively conserved in terms of core genome, ST73 appears more variable. Analysis of locally sequenced strains and comparison to globally-sourced reads from public databases can provide context which can allow the identification of outbreak clusters with more confidence than using total SNP counts alone and may help elucidate key outbreak groups and improve public health control of disease. This is valuable as the identification of clonal groups associated with outbreaks within larger bacterial populations remains a challenge.

Characterisation of molecular signatures can also assist in the identification of outbreaks as their transfer requires physical proximity of cells. CRL including integrons and transposons are common sites of genetic rearrangement and frequently carry unique molecular signatures due to insertion elements such as IS26 (Dawes et al., 2010; Roy Chowdhury et al., 2015; 
McKinnon et al., 2018). While all class 1 integrons in the Sydney collection are not necessarily novel, there are IS-mediated signature deletions which do not appear to have been widely reported based on current literature. This suggests that these are local integron variants, an idea consistent with the lack of these structures in the global SRA cohort. The major representative class 1 integron described here has been reported in its entirety once within an Australian E. coli O2:K1:H7 ST95 strain isolated from a bloodstream infection in 2010 (unpublished data; GenBank accession CP021289.1). This integron also shares an IS26mediated deletion of the Tn21 resolvase gene tnpR with plasmid pUO-SeVR1 from a Spanish Salmonella enterica serovar Enteritidis strain sourced from a child with gastroenteritis [49, 53]. This is significant as this precise signature is likely the product of a single event. As such, a lateral transfer event is a likely explanation for the occurrence of this signature in disparate and geographically separate strains followed by changes in class 1 integron cassette content. It is likely that transfer of these integrons is being facilitated by IncF plasmids similar to pUO-SeVR1, as this is the major plasmid incompatibility type within our ST73 collection and our S1-PFGE data confirm that the class 1 integrons described here are plasmid-borne. Plasmids appear to increasingly play an important role in the mobilisation of drug resistance genes in ExPEC ST73, and their characterization relies heavily on the use of whole-genome sequencing (ideally long-read) and read-mapping technologies such as those described here.

Whole genome sequencing allows for the analysis of gene presence/absence in clinical isolates which will provide data on the importance of virulence genes in pathogenesis. The virulence profiles of strains sequenced in this study are consistent with other examinations of virulence in ST73 and in ExPEC more broadly. Genes encoding P fimbrial adhesins, the aerobactin siderophore (iuc/iut), and toxins hemolysin A and cytotoxic necrotizing factor 1 are not universally identified in worldwide ExPEC populations sourced from humans and 
animals [1, 54-56]. In previous work on ST73 isolates sourced from the UK, the prevalence of these genes/gene families was also non-universal; however, hlyA and cnfl showed substantially higher prevalence in ST73 compared with ExPEC-associated ST10, ST69 and ST95 [1]. In isolates sourced from Sydney, a relatively clear association could be identified between phylogenetic groups and virulence profiles. Further in silico categorization of virulence profiles using global ST73 reads would provide insight into virulence patterns/groups within ST73 and ExPEC, which could potentially lead to improved response, prevention and treatment of ExPEC linked-disease.

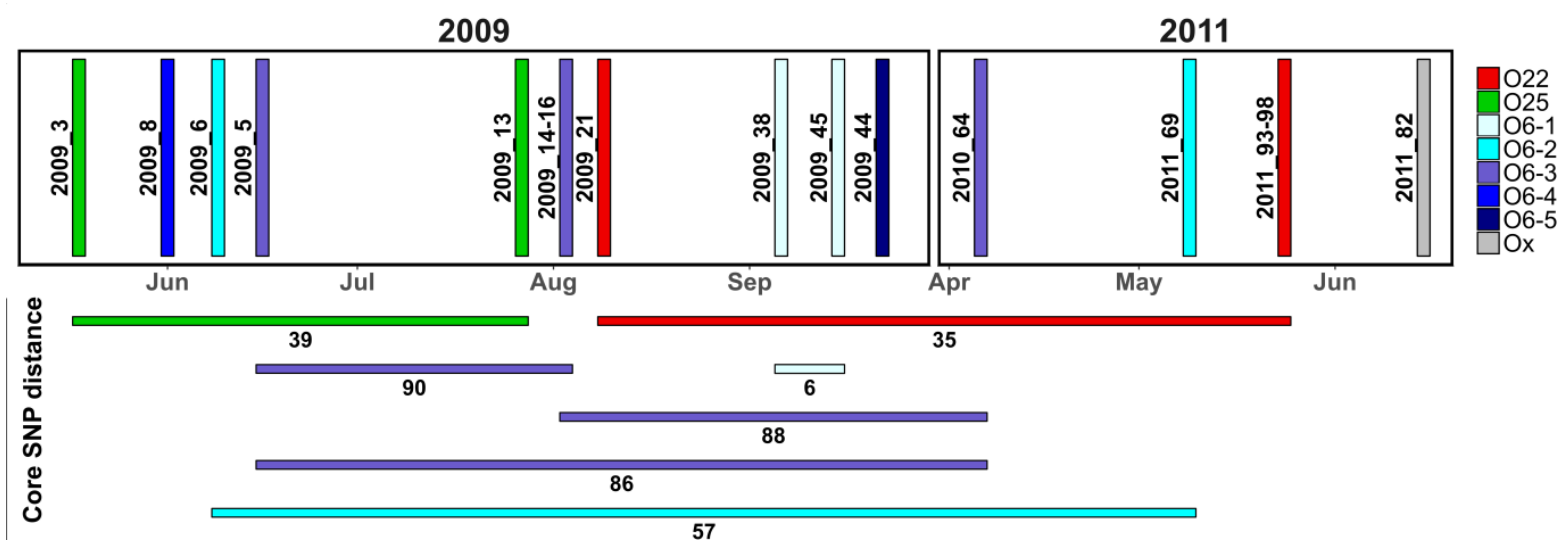

Figure 4. Epidemic curve for ST73 isolates sequenced in this study. Isolates are coloured to match groups identified by comparison to isolates from the Sequence Read Archive. Core SNP distances between samples of the same group are shown with coloured horizontal lines.

In endemic pathogens like E. coli, genetic comparisons of clonal group and mobile genetic element diversity can be difficult to perform with localized populations as high numbers of closely related isolates are required for robust SNP-phylogenetic analysis and this may require the long-term collection of bacterial isolates to isolate a sufficient number of representatives. Here we used Illumina sequencing combined with SNP-phylogenetic methods to identify at least eight distinct clonal lineages in a pilot sample of 16 ST73 isolates collected from a single hospital, indicating the wealth of diversity within ST73 population sourced from highly localised sampling over an extended period (Fig. 4). Contrastingly, the 
diversity of mobile elements within this cohort is much less profound. Only three resistance containing class 1 integron structures were identified, all were linked to plasmids, and all showed high structural similarity. Our study is an example of how genome sequencing can provide a depth of information not available with previous molecular epidemiology methodologies that are useful in the determination of outbreak groups among ST73 and the study of mobile genetic transfer in local populations.

\section{$\underline{\text { Author Statements }}$}

DB performed the phylogenetic analysis, contributed to gene presence analyses, prepared most of the Figures, Tables and supplementary data and drafted multiple iterations of the manuscript. JM isolated genomic DNA, contributed to gene presence analyses, characterised structures of class 1 integrons of Sydney strains as well as generated the figure and contributed to writing the manuscript. ML constructed libraries and sequenced the Sydney ST73 strains. CV and JI performed the S1-PGFE and southern blot analyses and edited final drafts of the manuscript. AD assisted with data analysis. PRC initiated the collaboration with Sydney Adventist Hospital, screened isolates to create the strain collection included in this study and helped JM with analysis of the data presented. SD initiated and coordinated the project and drafted iterations of the final manuscript.

\section{Funding information}

This work was supported by the Australian Research Council, Linkage Grant LP150100912. This project was partly funded by the Australian Centre for Genomic Epidemiological Microbiology (Ausgem), a collaborative partnership between the NSW Department of Primary Industries and the University of Technology Sydney. JM is a recipient of Australian Government Research Training Program Scholarships. 


\section{Acknowledgements}

We acknowledge the efforts of staff from the Sydney Adventist Hospital for providing the Sydney ST73 strains and associated metadata for this study.

\section{Ethical statement}

Ethics clearance was not required.

\section{Conflicts of interest}

There are no conflicts of interest to declare

\section{$\underline{\text { Abbreviations }}$}

ST

Sequence type

ExPEC Extraintestinal Escherichia coli

SNP Single nucleotide polymorphism

S1-PFGE S1 Nuclease - Pulsed Field Gel Electrophoresis

CS Conserved segments

UPEC Uropathogenic E. coli

NMEC Neonatal meningitis-causing E. coli

APEC Avian pathogenic E. coli

UTI Urinary tract infection

VAG Virulence-associated gene

MDR Multiple drug resistant

SAN Sydney Adventist Hospital

LB Luria-Bertani

AMP Ampicillin

AMC Amoxicillin-clavulanic acid

LEX Cephalexin 

CIP
Ciprofloxacin
GEN Gentamicin
NIT Nitrofurantoin
NOR Norfloxacin
TMP Trimethoprim

\section{$\underline{\text { Supplemental material }}$}

1. All sequencing reads and assemblies for isolates sequenced in this study have been submitted to the ENA Sequence Read Archive and GenBank, respectively. GenBank, SRA accession numbers and URLs are included in Table S1.

2. Scripts used for the analysis of SNP phylogeny have been deposited in Github; (URL - https://github.com/bogemad/snp_phylogeny)

3. Figure S1 has been deposited in Figshare; DOI: 10.6084/m9.figshare.5477449 (URL - https://figshare.com/s/b720dcd41ca1c11fdae2)

4. Figure S2 has been deposited in Figshare; DOI: 10.6084/m9.figshare.5477485 (URL - $\underline{\text { https://figshare.com/s/0835e6174bf9f026ff61) }}$

5. Table S1 has been deposited in Figshare; DOI: 10.6084/m9.figshare.5477461 (URL https://figshare.com/s/e6ea61f9dde79e9b18dd)

6. Table S2 has been deposited in Figshare; DOI: 10.6084/m9.figshare.5477464 (URL https://figshare.com/s/70de292aa98e806dd05f)

7. Table S3 has been deposited in Figshare; DOI: 10.6084/m9.figshare.5477467 (URL https://figshare.com/s/ddf02248b7f2d6f6ebe6)

8. Table S4 has been deposited in Figshare; DOI: 10.6084/m9.figshare.5477473 (URL https://figshare.com/s/b91bbc880eb31e5f90fc) 
9. Table S5 has been deposited in Figshare; DOI: 10.6084/m9.figshare.5477476 (URL https://figshare.com/s/3b8be84c33225bb32d78)

10. Table S6 has been deposited in Figshare; DOI: 10.6084/m9.figshare.5477479 (URL https://figshare.com/s/7ba306327343fdeca167)

11. Additional supplemental vcf files of total and core SNPs identified from the whole genome phylogeny of 226 isolates have been deposited in Figshare; Core SNP - DOI: 10.6084/m9.figshare.5477458 (URL

https://figshare.com/s/e033c384ecaleab880e7); Total SNP - DOI: 10.6084/m9.figshare.5477455 (URL - https://figshare.com/s/fd0233d6cca641d44bee)

\section{$\underline{\text { References }}$}

1. Gibreel TM, Dodgson AR, Cheesbrough J, Fox AJ, Bolton FJ, Upton M: Population structure, virulence potential and antibiotic susceptibility of uropathogenic Escherichia coli from Northwest England. J Antimicrob Chemother 2012, 67(2):346-356.

2. Kim S, Sung JY, Cho HH, Kwon KC, Koo SH: Characteristics of the Molecular Epidemiology of CTX-M-Producing Escherichia coli Isolated from a Tertiary Hospital in Daejeon, Korea. J Microbiol Biotechnol 2016, 26(9):1643-1649.

3. Manges AR, Johnson JR: Reservoirs of Extraintestinal Pathogenic Escherichia coli. Microbiol Spectr 2015, 3(5).

4. Olesen B, Scheutz F, Menard M, Skov MN, Kolmos HJ, Kuskowski MA, Johnson JR: Three-Decade Epidemiological Analysis of Escherichia coli O15:K52:H1. J Clin Microbiol 2009, 47(6):1857-1862. 
5. Yun KW, Kim DS, Kim W, Lim IS: Molecular typing of uropathogenic

Escherichia coli isolated from Korean children with urinary tract infection.

Korean Journal of Pediatrics 2015, 58(1):20-27.

6. Johnson JR, Thuras P, Johnston BD, Weissman SJ, Limaye AP, Riddell K, Scholes D, Tchesnokova V, Sokurenko E: The Pandemic H30 Subclone of Escherichia coli

Sequence Type 131 Is Associated With Persistent Infections and Adverse

Outcomes Independent From Its Multidrug Resistance and Associations With Compromised Hosts. Clin Infect Dis 2016, 62(12):1529-1536.

7. Pitout JD: Extraintestinal Pathogenic Escherichia coli: A Combination of Virulence with Antibiotic Resistance. Front Microbiol 2012, 3:9.

8. Wirth T, Falush D, Lan R, Colles F, Mensa P, Wieler LH, Karch H, Reeves PR, Maiden MC, Ochman $\mathrm{H}$ et al: Sex and virulence in Escherichia coli: an evolutionary perspective. Mol Microbiol 2006, 60(5):1136-1151.

9. Phan MD, Peters KM, Sarkar S, Lukowski SW, Allsopp LP, Gomes Moriel D, Achard ME, Totsika M, Marshall VM, Upton M et al: The serum resistome of a globally disseminated multidrug resistant uropathogenic Escherichia coli clone. PLoS Genet 2013, 9(10):e1003834.

10. de Kraker ME, Jarlier V, Monen JC, Heuer OE, van de Sande N, Grundmann H: The changing epidemiology of bacteraemias in Europe: trends from the European Antimicrobial Resistance Surveillance System. Clin Microbiol Infect 2013, 19(9):860-868.

11. Alhashash F, Wang X, Paszkiewicz K, Diggle M, Zong Z, McNally A: Increase in bacteraemia cases in the East Midlands region of the UK due to MDR 
Escherichia coli ST73: high levels of genomic and plasmid diversity in causative isolates. J Antimicrob Chemother 2016, 71(2):339-343.

12. de Souza da-Silva AP, de Sousa VS, Martins N, da Silva Dias RC, Bonelli RR, Riley LW, Moreira BM: Escherichia coli sequence type 73 as a cause of community acquired urinary tract infection in men and women in Rio de Janeiro, Brazil. Diagn Microbiol Infect Dis, 88(1):69-74.

13. Miajlovic H, Mac Aogáin M, Collins CJ, Rogers TR, Smith SGJ: Characterization of Escherichia coli bloodstream isolates associated with mortality. J Med Microbiol 2016, 65(1):71-79.

14. Yahiaoui M, Robin F, Bakour R, Hamidi M, Bonnet R, Messai Y: Antibiotic Resistance, Virulence, and Genetic Background of Community-Acquired Uropathogenic Escherichia coli from Algeria. Microb Drug Resist 2015, 21(5):516526.

15. Clermont O, Gordon D, Denamur E: Guide to the various phylogenetic classification schemes for Escherichia coli and the correspondence among schemes. Microbiology 2015, 161(Pt 5):980-988.

16. Riley LW: Pandemic lineages of extraintestinal pathogenic Escherichia coli. Clin Microbiol Infect 2014, 20(5):380-390.

17. Bengtsson S, Naseer U, Sundsfjord A, Kahlmeter G, Sundqvist M: Sequence types and plasmid carriage of uropathogenic Escherichia coli devoid of phenotypically detectable resistance. J Antimicrob Chemother 2012, 67(1):69-73. 
18. Kahlmeter G, Åhman J, Matuschek E: Antimicrobial Resistance of Escherichia coli Causing Uncomplicated Urinary Tract Infections: A European Update for 2014 and Comparison with 2000 and 2008. Infectious Diseases and Therapy 2015, 4(4):417-423.

19. Gillings MR, Gaze WH, Pruden A, Smalla K, Tiedje JM, Zhu YG: Using the class 1 integron-integrase gene as a proxy for anthropogenic pollution. ISME J 2015, 9(6):1269-1279.

20. Bell SM, Pham JN, Rafferty DL, Allerton JK: Antibiotic susceptibility tesing by the CDS method: A manual for medical and veterinary laboratories 2016, 8th edn. Kogarah, Australia: South Eastern Area Laboratory Services; 2016.

21. Coil D, Jospin G, Darling AE: A5-miseq: an updated pipeline to assemble microbial genomes from Illumina MiSeq data. Bioinformatics 2015, 31(4):587589.

22. Bankevich A, Nurk S, Antipov D, Gurevich AA, Dvorkin M, Kulikov AS, Lesin VM, Nikolenko SI, Pham S, Prjibelski AD et al: SPAdes: a new genome assembly algorithm and its applications to single-cell sequencing. J Comput Biol 2012, 19(5):455-477.

23. Inouye M, Dashnow H, Raven LA, Schultz MB, Pope BJ, Tomita T, Zobel J, Holt KE: SRST2: Rapid genomic surveillance for public health and hospital microbiology labs. Genome Med 2014, 6(11):90.

24. Chen L, Yang J, Yu J, Yao Z, Sun L, Shen Y, Jin Q: VFDB: a reference database for bacterial virulence factors. Nucleic Acids Res 2005, 33(Database issue):D325328. 
25. Gupta SK, Padmanabhan BR, Diene SM, Lopez-Rojas R, Kempf M, Landraud L, Rolain JM: ARG-ANNOT, a new bioinformatic tool to discover antibiotic resistance genes in bacterial genomes. Antimicrob Agents Chemother 2014, 58(1):212-220.

26. Minogue TD, Daligault HA, Davenport KW, Bishop-Lilly KA, Broomall SM, Bruce DC, Chain PS, Chertkov O, Coyne SR, Freitas T et al: Complete Genome Assembly of Escherichia coli ATCC 25922, a Serotype O6 Reference Strain. Genome Announc 2014, 2(5):e00969-00914.

27. Reeves PR, Liu B, Zhou Z, Li D, Guo D, Ren Y, Clabots C, Lan R, Johnson JR, Wang L: Rates of Mutation and Host Transmission for an Escherichia coli Clone over 3 Years. PLoS One 2011, 6(10):e26907.

28. Reister M, Hoffmeier K, Krezdorn N, Rotter B, Liang C, Rund S, Dandekar T, Sonnenborn U, Oelschlaeger TA: Complete genome sequence of the gram-negative probiotic Escherichia coli strain Nissle 1917. J Biotechnol 2014, 187:106-107.

29. Zdziarski J, Brzuszkiewicz E, Wullt B, Liesegang H, Biran D, Voigt B, GronbergHernandez J, Ragnarsdottir B, Hecker M, Ron EZ et al: Host imprints on bacterial genomes--rapid, divergent evolution in individual patients. PLoS Pathog 2010, 6(8):e1001078.

30. Welch RA, Burland V, Plunkett G, 3rd, Redford P, Roesch P, Rasko D, Buckles EL, Liou SR, Boutin A, Hackett J et al: Extensive mosaic structure revealed by the complete genome sequence of uropathogenic Escherichia coli. Proc Natl Acad Sci U S A 2002, 99(26):17020-17024. 
31. Dallman TJ, Byrne L, Ashton PM, Cowley LA, Perry NT, Adak G, Petrovska L, Ellis RJ, Elson R, Underwood A et al: Whole-genome sequencing for national surveillance of Shiga toxin-producing Escherichia coli 0157. Clin Infect Dis 2015, 61(3):305-312.

32. Dunitz MI, Coil DA, Jospin G, Eisen JA, Adams JY: Draft Genome Sequences of Escherichia coli Strains Isolated from Septic Patients. Genome Announc 2014, 2(6).

33. Earle SG, Wu CH, Charlesworth J, Stoesser N, Gordon NC, Walker TM, Spencer CC, Iqbal Z, Clifton DA, Hopkins KL et al: Identifying lineage effects when controlling for population structure improves power in bacterial association studies. Nat Microbiol 2016, 1:16041.

34. Kluytmans-van den Bergh MF, Rossen JW, Bruijning-Verhagen PC, Bonten MJ, Friedrich AW, Vandenbroucke-Grauls CM, Willems RJ, Kluytmans JA: WholeGenome Multilocus Sequence Typing of Extended-Spectrum-Beta-LactamaseProducing Enterobacteriaceae. J Clin Microbiol 2016, 54(12):2919-2927.

35. Pesesky MW, Hussain T, Wallace M, Wang B, Andleeb S, Burnham CA, Dantas G: KPC and NDM-1 genes in related Enterobacteriaceae strains and plasmids from Pakistan and the United States. Emerg Infect Dis 2015, 21(6):1034-1037.

36. Roach DJ, Burton JN, Lee C, Stackhouse B, Butler-Wu SM, Cookson BT, Shendure J, Salipante SJ: A Year of Infection in the Intensive Care Unit: Prospective Whole Genome Sequencing of Bacterial Clinical Isolates Reveals Cryptic Transmissions and Novel Microbiota. PLoS Genet 2015, 11(7):e1005413. 
37. Salipante SJ, Roach DJ, Kitzman JO, Snyder MW, Stackhouse B, Butler-Wu SM, Lee C, Cookson BT, Shendure J: Large-scale genomic sequencing of extraintestinal pathogenic Escherichia coli strains. Genome Res 2015, 25(1):119-128.

38. Toro M, Cao G, Rump L, Nagaraja TG, Meng J, Gonzalez-Escalona N: Genome Sequences of 64 Non-O157:H7 Shiga Toxin-Producing Escherichia coli Strains. Genome Announc 2015, 3(5).

39. Barton BM, Harding GP, Zuccarelli AJ: A general method for detecting and sizing large plasmids. Anal Biochem 1995, 226(2):235-240.

40. Partridge SR, Zong Z, Iredell JR: Recombination in IS26 and Tn2 in the evolution of multiresistance regions carrying blaCTX-M-15 on conjugative IncF plasmids from Escherichia coli. Antimicrob Agents Chemother 2011, 55(11):4971-4978.

41. Koeleman JG, Stoof J, Van Der Bijl MW, Vandenbroucke-Grauls CM, Savelkoul PH: Identification of epidemic strains of Acinetobacter baumannii by integrase gene PCR. J Clin Microbiol 2001, 39(1):8-13.

42. Darling AE, Jospin G, Lowe E, Matsen FAt, Bik HM, Eisen JA: PhyloSift: phylogenetic analysis of genomes and metagenomes. PeerJ 2014, 2:e243.

43. Croucher NJ, Harris SR, Fraser C, Quail MA, Burton J, van der Linden M, McGee L, von Gottberg A, Song JH, Ko KS et al: Rapid pneumococcal evolution in response to clinical interventions. Science 2011, 331(6016):430-434.

44. Stamatakis A: RAxML version 8: a tool for phylogenetic analysis and postanalysis of large phylogenies. Bioinformatics 2014, 30(9):1312-1313. 
45. Nesta B, Spraggon G, Alteri C, Moriel DG, Rosini R, Veggi D, Smith S, Bertoldi I, Pastorello I, Ferlenghi I et al: FdeC, a novel broadly conserved Escherichia coli adhesin eliciting protection against urinary tract infections. MBio 2012, 3(2).

46. Ladomersky E, Petris MJ: Copper tolerance and virulence in bacteria. Metallomics 2015, 7(6):957-964.

47. Baker-Austin C, Wright MS, Stepanauskas R, McArthur JV: Co-selection of antibiotic and metal resistance. Trends Microbiol 2006, 14(4):176-182.

48. Nakaya R, Nakamura A, Murata Y: Resistance transfer agents in Shigella. Biochem Biophys Res Commun 1960, 3:654-659.

49. Garcia V, Garcia P, Rodriguez I, Rodicio R, Rodicio MR: The role of IS26 in evolution of a derivative of the virulence plasmid of Salmonella enterica serovar Enteritidis which confers multiple drug resistance. Infect Genet Evol 2016, 45:246-249.

50. Broom JE, Hill DF, Hughes G, Jones WA, McNaughton JC, Stockwell PA, Petersen GB: Sequence of a transposon identified as Tn1000 (gamma delta). DNA Seq 1995, 5(3):185-189.

51. Petty NK, Ben Zakour NL, Stanton-Cook M, Skippington E, Totsika M, Forde BM, Phan MD, Gomes Moriel D, Peters KM, Davies M et al: Global dissemination of a multidrug resistant Escherichia coli clone. Proc Natl Acad Sci U S A 2014, 111(15):5694-5699.

52. Price LB, Johnson JR, Aziz M, Clabots C, Johnston B, Tchesnokova V, Nordstrom L, Billig M, Chattopadhyay S, Stegger M et al: The Epidemic of Extended-Spectrum- 


\section{$\beta$-Lactamase-Producing Escherichia coli ST131 Is Driven by a Single Highly}

Pathogenic Subclone, H30-Rx. mBio 2013, 4(6):e00377-00313.

53. Rodriguez I, Rodicio MR, Herrera-Leon S, Echeita A, Mendoza MC: Class 1 integrons in multidrug-resistant non-typhoidal Salmonella enterica isolated in Spain between 2002 and 2004. Int J Antimicrob Agents 2008, 32(2):158-164.

54. Cunha MPV, Saidenberg AB, Moreno AM, Ferreira AJP, Vieira MAM, Gomes TAT, Knöbl T: Pandemic extra-intestinal pathogenic Escherichia coli (ExPEC) clonal group O6-B2-ST73 as a cause of avian colibacillosis in Brazil. PLoS One 2017, 12(6):e0178970.

55. Liu X, Thungrat K, Boothe DM: Multilocus Sequence Typing and Virulence Profiles in Uropathogenic Escherichia coli Isolated from Cats in the United States. PLoS One 2015, 10(11):e0143335.

56. Yun KW, Kim HY, Park HK, Kim W, Lim IS: Virulence factors of uropathogenic Escherichia coli of urinary tract infections and asymptomatic bacteriuria in children. J Microbiol Immunol Infect 2014, 47(6):455-461. 


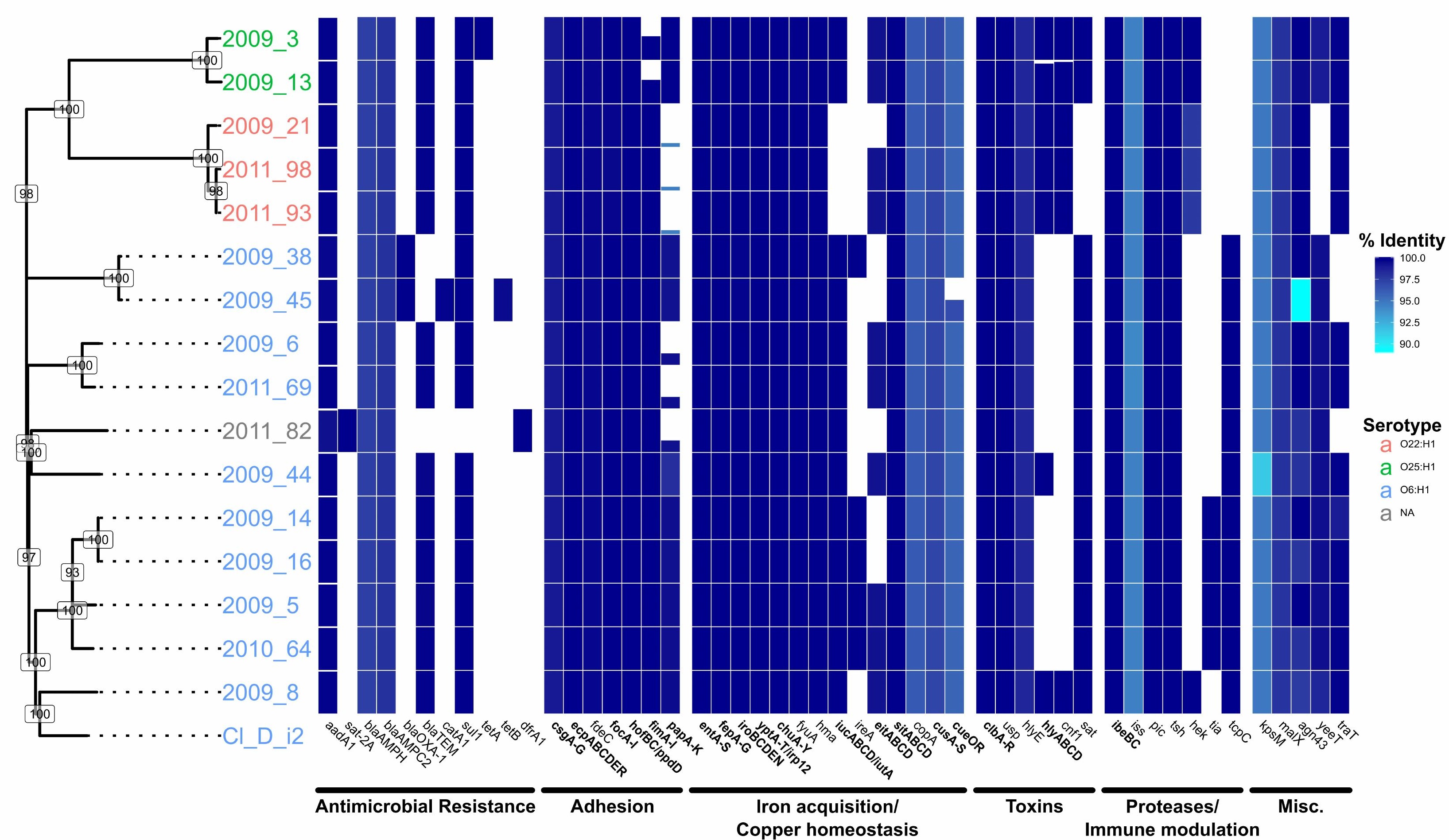




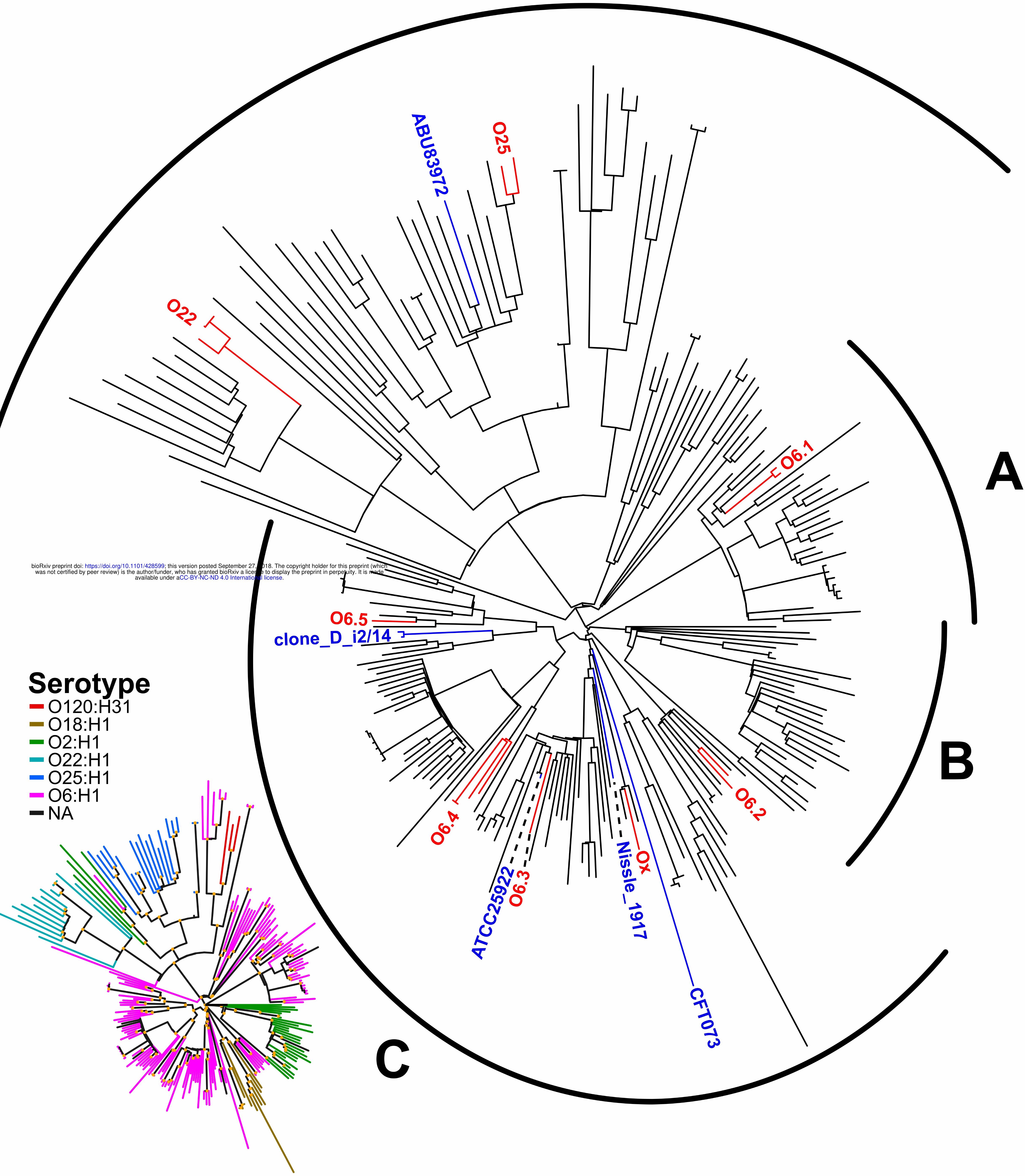




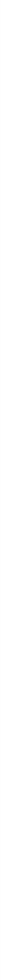

